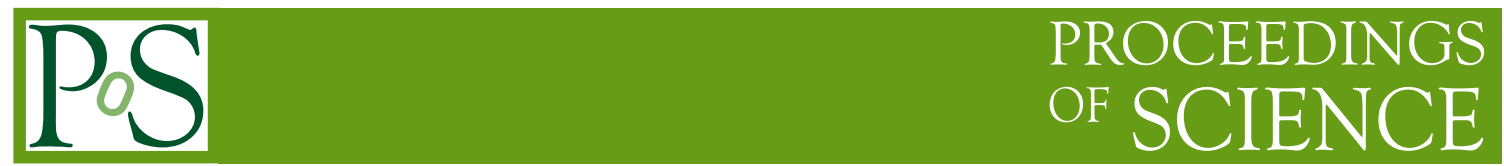

\title{
The Search for neutrinoless double beta-decay
}

\section{B. Majorovits*}

Max-Planck-Institut für Physik, München, Germany

E-mail: bela@mpp.mpg.de

Observation of neutrinoless double beta decay would greatly enlarge our knowledge on neutrino properties. It would reveal the Majorana nature of neutrinos and could yield information on their mass hierarchy. Experimental strategies to search for neutrinoless double beta-decay experiments are explained. The experiments with highest sensitivity in the past are discussed. The current status of experiments likely to deliver competitive data within the next two years are presented.

25th Texas Symposium on Relativistic Astrophysics - TEXAS 2010

December 06-10, 2010

Heidelberg, Germany

* Speaker. 


\section{Introduction}

Neutrinos are, after photons, the second most abundant known particles in the universe. Nevertheless there are many unknowns regarding their properties. Since the observation of neutrino oscillations [1] it is known that at least two neutrino mass eigenvalues are non zero. However, it is not known what the absolute mass scale of the spectrum is. Also the hierarchy of the mass eigenstates is unknown: It is unknown whether the mass eigenstate mostly associated to the electron neutrino is the lightest one. A key question regarding neutrino properties is whether or not they are their own anti-particles.

Observation of neutrinoless double beta-decay ( $0 v \beta \beta$-decay) could answer some of the abovementioned questions. $0 v \beta \beta$-decay can only occur for a non vanishing Majorana component of the neutrino mass [2]. The helicity flip in the vertex of the decay could be induced by a non vanishing neutrino mass. In this case the decay rate $\mathrm{R}$ is coupled to the effective Majorana neutrino mass

$$
<m_{e e}>=\left.\left|\Sigma_{i}\right| U_{e i}\right|^{2} e^{i \beta_{i}} m_{i} \mid
$$

by the relation

$$
R=G(Q) \cdot M_{n u c l}^{2} \cdot<m_{e e}>^{2},
$$

where $U_{e i}$ are the electron neutrino mixing matrix elements in of the neutrino mixing matrix, $e^{i \beta_{i}}$ are the complex phases factors in the matrix, $m_{i}$ are the masses eigenvalues, $G(Q)$ is the phase space factor of the decay that can be precisely calculated and $M_{n u c l}^{2}$ is the nuclear matrix element of the decay.

Other beyond standard model processes could also contribute to a non vanishing decay rate.

Observing a Majorana neutrino mass $\geq 0.1 \mathrm{meV}$ would imply an inverse neutrino mass hierarchy, assuming the mass mechanism is the dominating one [3].

\section{Experimental considerations}

During the process of $0 v \beta \beta$-decay no neutrinos are emitted. The complete energy released can in principle be detected. $0 v \beta \beta$-experiments search for a peak at the Q-value of the decay. For an effective neutrino mass $\leq 0.2 \mathrm{eV}$ the expected half life for ${ }^{76} \mathrm{Ge}$, a double beta emitter, are $T_{1 / 2}^{0 v \beta \beta} \geq 10^{25}$ years.

A figure of merit for the sensitivity of an experiment with non vanishing background searching for rare events can be introduced:

$$
\text { F.O.M. }=M_{\text {nucl }} \cdot \varepsilon \cdot a \cdot \sqrt{\frac{m \cdot T}{b \cdot F W H M}},
$$

where $\varepsilon$ is the detection efficiency, $a$ the abundance of the double beta emitter in the detector, $m$ the active detector mass, $T$ the measuring time, $b$ the background index and $F W H M$ the energy window used for the analysis usually proportional to the energy resolution of the detector. For an experiment without background this expression changes to

$$
\text { F.O.M }=M_{\text {nucl }} \cdot \varepsilon \cdot a \cdot m \cdot T \text {. }
$$


Table 1: Selective list of experiments performed with different isotopes using different technologies in different underground labs. Listed are the results in form of half-life limits and the range of limits on the effective Majorana neutrino mass using different nuclear matrix element calculations. These values have to be interpreted with caution.

\begin{tabular}{|c|c|c|c|c|c|}
\hline Experiment & Underground lab & Isotope & Technology & $\begin{array}{l}\mathrm{T}_{1 / 2} \text { limit } \\
{\left[10^{24} \mathrm{y}\right]}\end{array}$ & $\begin{array}{l}\text { mass limit } \\
{[\mathrm{eV}]}\end{array}$ \\
\hline Elegant VI & Oto (Japan) & ${ }^{48} \mathrm{Ca}$ & Scintillator & $>0.058$ & $<3.5-22$ \\
\hline Heidelberg-Moscow & LNGS (Italy) & ${ }^{76} \mathrm{Ge}$ & HPGe & $>19$ & $<0.35-1.2$ \\
\hline Subgroup of HdMo & & & & $22.3_{-3.1}^{+4.4}$ & $0.28_{-0.11}^{+0.17}$ \\
\hline IGEX & Canfranc (Spain) & ${ }^{76} \mathrm{Ge}$ & HPGe & $>16$ & $<0.3-1.5$ \\
\hline NEMO III & Frejus (France) & ${ }^{100} \mathrm{Mo}$ & Tracker & $>0.31$ & $<0.8-1.2$ \\
\hline $\mathrm{CdWO}_{4}$ & Solotvina (Ukrain) & ${ }^{116} \mathrm{Cd}$ & Light & $>0.17$ & $<1.5-1.7$ \\
\hline CUORECINO & LNGS (Itlay) & ${ }^{130} \mathrm{Te}$ & Bolometer & $\geq 2.8$ & $\leq 0.3-0.7$ \\
\hline
\end{tabular}

A "hypothetically ideal" $0 v \beta \beta$-decay experiment will thus use a background free technology with ideal energy resolution where the detector is built from the source isotope $(\varepsilon \approx 1)$ and uses huge quantities of a financially available isotope with $100 \%$ natural abundance with the highest possible $M_{\text {nucl }}$.

\section{Results from former experiments}

In reality the choice of isotope determines most parameters: Elements with low abundance of the double beta emitting isotope can be artificially enriched. This can be cost prohibitive. The detection efficiency $\varepsilon$ is limited to quantities $\leq 1$ and will be highest for isotopes from which detectors can be built (source=detector). Some double beta emitting isotopes such as ${ }^{76} \mathrm{Ge}$ or ${ }^{130} \mathrm{Te}$ exist out of which high resolution detectors can be built. The $F W H M$ will depend on the isotope as its choice determines the possible methods for radiation detection. The best achieved FWHM at the energy region of interest is around $0.2 \%$ for germanium detectors. Bolometric approaches have achieved energy resolutions of around $0.25 \%$ at the energy of interest, not far behind HPGe detector technology. $M_{\text {nucl }}$ is given by the isotope and varies between the isotopes with reasonable Q-values by a factor of $\approx 2.5$. Thus, once the isotope and the technology are chosen, the parameters that can experimentally be influenced to increase the sensitivity are the background index $b$ and the exposure $m \cdot T$. For some technologies also the energy resolution has not yet reached its physical limits.

To minimize the background $b$ from the direct surrounding, three approaches are followed:

- Minimize materials in the close surrounding of the detector. Any infra-structural material is a potential source for background radiation.

- Use only selected high radio-purity materials.

- Build intelligent detectors that can distinguish background from the signal. In $0 v \beta \beta$ events the energy is typically deposited within a few millimeters. Background $\gamma_{\mathrm{s}}$ of $2 \mathrm{MeV}$ have a mean free path in germanium of a few centimeters. Thus it is rather likely that a background 
Table 2: List of $0 v \beta \beta$-experiments that have the sensitivity to test the evidence for $0 v \beta \beta$-decay [8] and are in or close to their commissioning phase. Sensitivities on the effective Majorana mass depend on the nuclear matrix element calculations used.

\begin{tabular}{ccccccc}
\hline Experiment & Isotope & $\begin{array}{c}\text { Target } \\
\text { mass }\end{array}$ & Technology & $\begin{array}{c}\text { Energy } \\
\text { resolution }\end{array}$ & $\begin{array}{c}\text { Sensitivity } \\
{[\mathrm{meV}]}\end{array}$ & $\begin{array}{c}\text { Planned } \\
\text { start }\end{array}$ \\
\hline \hline GERDA I & ${ }^{76} \mathrm{Ge}$ & 18 & HPGe & $0.20 \%$ FWHM & $\begin{array}{c}220-500 \\
90-200\end{array}$ & 2011 \\
GERDA II & & 40 & & & & 2012 \\
\hline Majorana & ${ }^{76} \mathrm{Ge}$ & $\approx 20$ & $\mathrm{HPGe}^{2}$ & $0.20 \%$ FWHM & & 2012 \\
\hline CUORE0 & ${ }^{130} \mathrm{Te}$ & 10 & $\mathrm{TeO}_{2}$ & $0.25 \%$ FWHM & $168-391$ & 2011 \\
CUORE & & 200 & bolometer & & $41-96$ & 2013 \\
\hline EXO & ${ }^{136} \mathrm{Xe}$ & 100 & LXe TPC & $1.6 \% 1 \sigma$ & $130-190$ & 2011 \\
\hline
\end{tabular}

$\gamma$ deposits its energy within a much larger volume. If the event topology can be reconstructed a good fraction of background events can be identified.

All low background experiments nowadays are built in underground laboratories to avoid the cosmic radiation and are shielded against the natural radio-activity in the surrounding of the underground laboratory.

Results from some selected former experiments are shown in Table 11. The HPGe HeidlebergMoscow [ [ $₫$ ] and IGEX [河] experiments together with the results from the CUORICINO [6] give the most stringent limits on the half life of $0 v \beta \beta$-decay. A subgroup of the Heidelberg-Moscow experiment published evidence for observation of $0 v \beta \beta$-decay with a half-life of $2.23_{-0.31}^{+0.44} \cdot 10^{25}$ years [8]. This corresponds to an effevtive Majorana neutrino mass of $0.35 \mathrm{ev}-1.2 \mathrm{eV}$ depending on the used matrix element calculation.

\section{Status of new approaches}

Several new experiments with expected sensitivities sufficient to test the Heidelberg-Moscow evidence are about to start data taking and commissioning. They are summarized in Table 2.

\subsection{GERDA}

The conceptual design of the GERDA experiment [11] bases on the idea of using an ultra-pure cryogenic liquid as the cooling medium and as a shield against gamma radiation simultaneously [12]. A cryostat (see Fig 1 left) made out of carefully selected stainless steel houses an array of HPGe detectors. The cryostat has a low-background copper inset as a radiation shield against the steel of the cryo-tank. The cryogenic volume is surrounded by a buffer of ultra-pure water acting as an additional gamma and neutron shield. The water buffer is additionally used as a muon-Čerenkov veto. The setup is schematically depicted in Fig. 1.

The whole GERDA infrastructure has been completed in May 2010 in hall A of the LNGS underground laboratory in Italy. A string of three detectors with a total mass of $7.6 \mathrm{~kg}$ (see Fig. 1 right) is taking background data since June 2010. Calibration of the detectors showed energy resolutions of these detectors between $4 \mathrm{keV}$ and $5 \mathrm{keV}$ (see Fig. \&, left). 


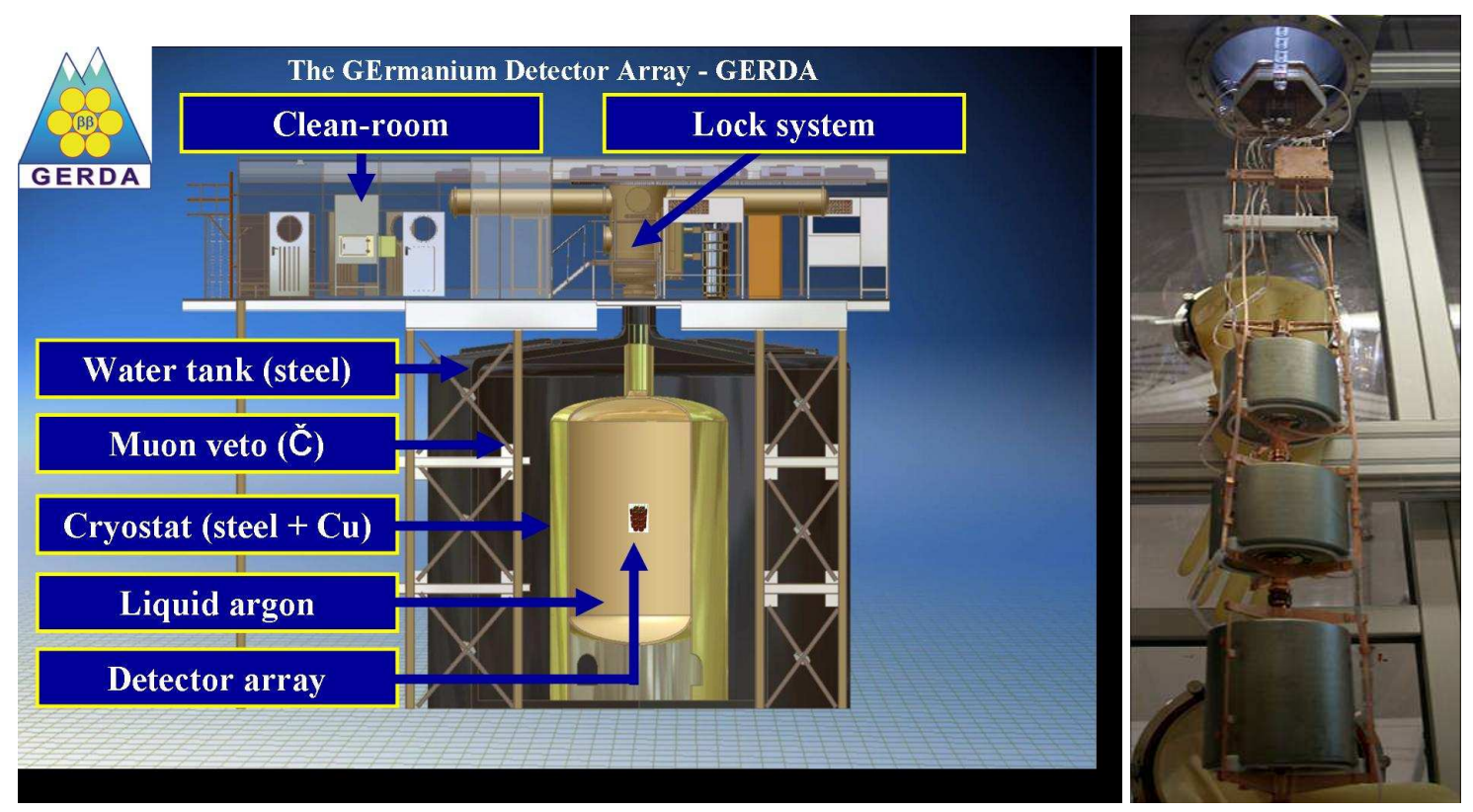

Figure 1: Left: Sketch of the GERDA experiment.

The background in the energy region of interest around $2 \mathrm{MeV}$ is better than in the HeidelbergMoscow and IGEX experiments but is presently not at the design level of $10^{-2} \mathrm{cts} /(\mathrm{kg} \mathrm{y} \mathrm{keV})$. A prominent peak in the spectrum at $1525 \mathrm{keV}$ (see Fig. 目 right) is attributed to the decay of ${ }^{42} \mathrm{~K}$. It is the daughter isotope of ${ }^{42} \mathrm{Ar}$, which is a beta- emitter with a Q-value of $600 \mathrm{keV}$ and a half life of 33 years. Its origin in the liquid argon is not yet understood. The daughter isotope ${ }^{42} \mathrm{~K}$ is a betaemitter with a Q-value of $3.5 \mathrm{MeV}$ and it decays with a half life of 12.4 hours with $82 \%$ probability to the ground state of ${ }^{42} \mathrm{Ca}$. With $17.6 \%$ probability ${ }^{42} \mathrm{~K}$ decays into an excited state of ${ }^{42} \mathrm{Ca}$ at the energy level of $1525 \mathrm{keV}$. In this case the excited ${ }^{42} \mathrm{Ca}$ nucleus emits a $1525.5 \mathrm{keV}$ gamma.

After the decay of ${ }^{42} \mathrm{Ar}$ a positive ${ }^{42} \mathrm{~K}$ ion remains. The life time of ions in liquid argon can be long. Thus if an electric field is present, charged ions can drift. As the detectors are biased and thus create an electric field, it is possible that charged ions drift to the detector before they neutralize and decay. In this case the ${ }^{42} \mathrm{~K}$ beta- decay could happen in the direct vicinity of the detector. This is one possible explanation for the background above the peak seen in Fig. 2 right. This would, however, also imply that by changing the electric field the influence of this background can be controlled. Presently these possibilities are being investigated within the GERDA setup.

In the first phase, enriched detectors which were previously operated by the HeidelbergMoscow and IGEX collaborations will be re-deployed after re-processing. If the HeidelbergMoscow claim is correct GERDA can either confirm or refute the claim from the HdM experiment within one year of measuring time [15].

In the second phase custom made detectors will be installed. Detectors with a special electrode configuration, so called BEGe detectors will be used. These type of detectors allow for event topology reconstruction by pulse shape analysis [13] and thus for identification of Compton-scattered $\gamma \mathrm{s}$. To test technologies for use in a one ton experiment segmented detectors are also discussed [14]. An exposure of $100 \mathrm{~kg} \cdot \mathrm{y}$ with a background level of $10^{-3} \mathrm{cts} /(\mathrm{keV} \mathrm{kg} \mathrm{y})$ is foreseen. From this a lower limit on the half-life of $0 v \beta \beta$-decay of higher than $1.5 \cdot 10^{26} \mathrm{y}$ can be set with $90 \%$ probability 

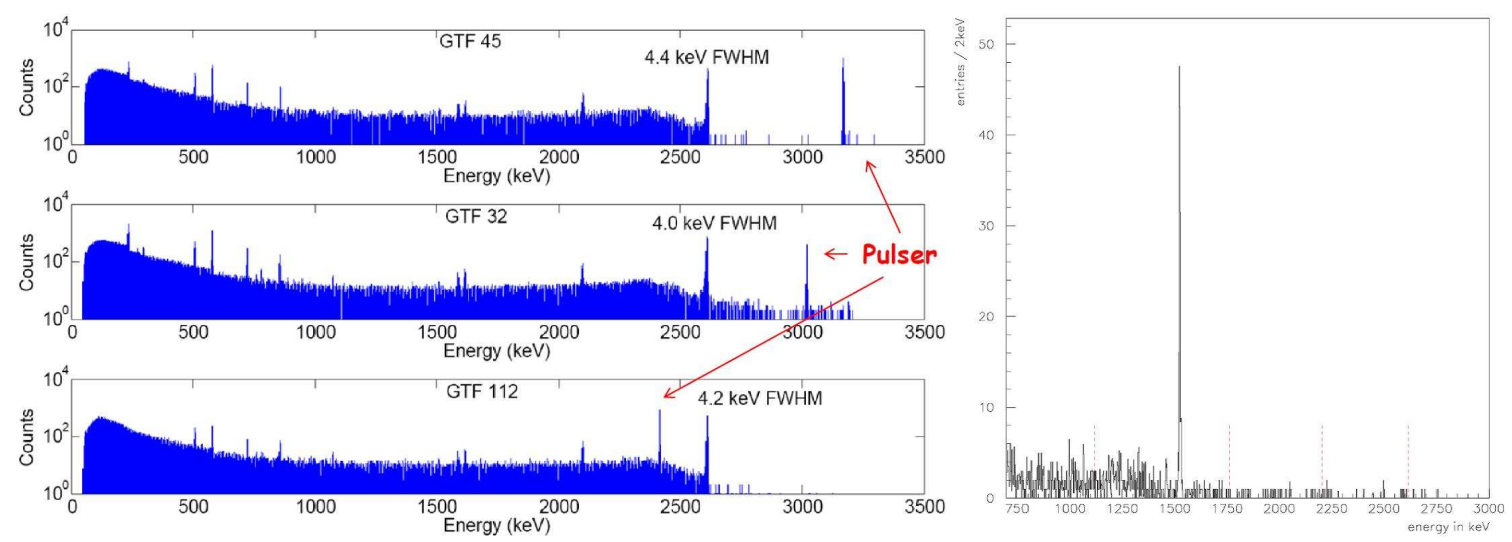

Figure 2: Calibration (left) and a background spectrum (right) taken with a non enriched HPGe detector. The dashed lines mark the energies where lines from natural background might be expected.

if no signal is observed [15].

\subsection{Majorana}

The design concept of the Majorana experiment[16] is to use HPGe BEGe detectors enriched to $86 \%$ in ${ }^{76} \mathrm{Ge}$ a conventional vacuum cryostat made of specially electro-formed copper that can be produced with very high purity [16]. This material will also be used for the shielding. Like phase II of GERDA, $20 \mathrm{~kg}$ of natural BEGe HPGe detectors will be used in the first phase. Commissioning at the DUSEL underground laboratory is scheduled to start in 2012. In 2013 an additional $20 \mathrm{~kg}$ of BEGe HPGe detectors enriched in ${ }^{76} \mathrm{Ge}$ are scheduled to be included

\subsection{CUORE}

Crystals made from ${ }^{\text {nat }} \mathrm{TeO}_{2}$ will be used in the CUORE experiment [17] at the LNGS underground laboratory. These are cooled to $\mathrm{mK}$ temperatures. Energy deposits are measured by detecting the temperature increase of the crystal. In a $0^{\text {th }}$ phase the old Cuoricino [6] cryostat will be used. A detector tower with $52 \mathrm{TeO}_{2}$ crystals with improved radio-purity will be installed to improve the background index of $0.16 \pm 0.01 \mathrm{cts} /(\mathrm{kg} \mathrm{y} \mathrm{keV})$ reached in the Cuoricine experiment to $0.12 \mathrm{cts} /(\mathrm{kg} \mathrm{y} \mathrm{keV})[6]$. The experiment will house $15 \mathrm{~kg}$ of the doube beta-emitting isotope ${ }^{130} \mathrm{Te}$. With this assumptions a sensitivity of the experiment to the half live of ${ }^{130} \mathrm{Te}$ of $8 \cdot 10^{24} \mathrm{y}$ could be reached within two years of measurement. Commissioning is scheduled to start in late 2011. A new cryostat is meanwhile being built that will be able to house up to $988 \mathrm{TeO}_{2}$ crystals containing a total mass of $200 \mathrm{~kg}$ of the isotope ${ }^{130} \mathrm{Te}$. With a background index of $10^{-2} \mathrm{cts} /(\mathrm{kg} \mathrm{y} \mathrm{keV})$ in the energy region of interest and an energy resolution of $0.25 \%$, a sensitivity is expected that would exclude Majorana masses above $41 \mathrm{eV}-96 \mathrm{eV}$ Commissioning of this phase is planned for 2013.

\subsection{EXO}

The EXO experiment [18] uses $200 \mathrm{~kg}$ of liquid xenon enriched to $80 \%$ in ${ }^{136} \mathrm{Xe}$. It will be operated as a Time Projection Chamber (TPC), in which scintillation of liquid xenon will be detected using avalanche photo-diodes while ionization electrons can be detected by grid wires. 
The drift time of the electrons depends on the location of the interaction, thus the event vertex can be reconstructed. The active volume of the detector contains $70 \%$ of the total mass. The expected energy resolution of $1.6 \%(1 \sigma)$ would allow for a sensitivity to probe the Majoran neutrino mass down to $130 \mathrm{eV}-190 \mathrm{eV}$. Commissioning of the EXO experiment has started with non enriched liquid xenon. First results with enriched Xenon are expected for 2011.

\section{Conclusion}

Neutrinoless double beta-decay can probe the Majorana nature of neutrinos. The past generation of experiments has set limits on the effective Majorana neutrino mass in the range of few hundred meV. A subgroup of the Heidelberg-Moscow collaboration claims evidence for observation of neutrinoless double beta-decay in ${ }^{76} \mathrm{Ge}$. This evidence has to be tested with ${ }^{76} \mathrm{Ge}$ but also independently with different isotopes. The GERDA, Majorana, CUORE and EXO experiments are presently under commissioning or will start data taking soon.

\section{References}

[1] S. N. Ahmed et al. SNO Collaboration, Phys. Rev. Lett. 92(2004)181301

[2] J. Schechter, J. Valle, Phys. Rev. D 25(1982)2951

[3] F. Feruglio et al., Nucl. Phys. B 637(2002)345

[4] H.V. Klapdor-Kleingrothaus et al., Eur. Phys. J. A 12(2001)147

[5] C.E. Aalseth et al., Phys. Rev. D 65(2002)092007 e-Print: hep-ex/0202026

[6] M. Pavan, presentation at Neutrino 2010, Athens, http://www.neutrino2010.gr, to be published at Nucle. Phys. B Proc. Suppl.

[7] S. Umehara et al., Phys. Rev. C 78(2008)058501

[8] H.V. Klapdor-Kleingrothaus et al., Mod. Phys. Lett. A 21(2006)1547

[9] R. Arnold et al., JETP Lett. 80(2004)377, Pisma Zh. Eksp. Teor. Fiz. 80(2004)429, e-Print: hep-ex/0410021

[10] F.A. Danevich et al., Phys. Rev .C 68(2003)035501

[11] GERDA collaboration, I. Abt et al., arXiv:hep-ex/0404039 and S. Schönert et al., Nucl. Phys. Proc. Suppl. 145(2005)242

[12] G. Heusser, Ann. Rev. Nucl. Part. Sci. 45 (1995)543

[13] M.B. Heider et al., J. Instrum. 5(2010)p10007

[14] I. Abt et al., Eur. Phys. J. C 52(2007)19-27

[15] A. Caldwell and K. Kröninger, Phys. Rev. D 74(2006)092003

[16] The Majorana collaboration, arXiv:nucl-ex/0311013

[17] CUORE proposal, R.Ardito et al.,arXiv:hep-ex/0501010

[18] M. Danilov et al., Phys. Lett. B 480(2000)12 